\title{
A VIA VIDEOLAPAROSCÓPICA PODE SER UTILIZADA COMO ROTINA NAS ESPLENECTOMIAS?
}

\author{
Can videolaparoscopic access be used as routine in splenectomies?
}

\author{
Eduardo Neubarth TRINDADE, Manoel Roberto Maciel TRINDADE, Ricardo FRANCIO, Elisa Pedrebon ZANELLA
}

Trabalho realizado no Serviço de Cirurgia Digestiva do Hospital de Clínicas de Porto Alegre e no Departamento de Cirurgia da Universidade Federal do Rio Grande do Sul, Porto Alegre, RS, Brasil.

DESCRITORES - Esplenectomia. Baço.
RESUMO - Racional - A esplenectomia laparoscópica é alternativa para o tratamento de pacientes submetidos à esplenectomia eletiva. Uma de suas principais indicações está nas doenças hematológicas que não respondem ao tratamento clínico. A videolaparoscopia apresenta vantagens para os pacientes, quando comparados à laparotomia: menos dor no pós-operatório, recuperação mais célere das funções do trato gastrointestinal, melhores resultados estéticos e menor tempo de hospitalização. Objetivo - Apresentar série de casos de esplenectomia videolaparoscópica de um hospital universitário. Métodos - Forram avaliadas as esplenectomias realizadas entre junho de 2005 e outubro de 2012. A análise foi prospectiva dividida em pré, trans e pós-operatórios dos seguintes dados: gênero, idade, indicação da operação, taxa de conversão para laparotomia, duração do procedimento, tamanho do baço, presença de baço acessório, tempo de internação e resposta em oito semanas do pós-operatório. Resultados - Foram analisadas 44 esplenectomias laparoscópicas realizadas no período. Os pacientes foram com púrpura trombocitopênica idiopática sem resposta ao tratamento farmacológico representaram 56,8\%; anemia hemolítica auto-imune foi de 13,6\%; esferocitose de $11,3 \%$ e 18,3\% por outras causas não-hemolíticas. 0 tempo cirúrgico médio foi de 166,7 (60-319) minutos. Apenas quatro pacientes $(9,1 \%)$ tiveram complicações pós-operatórias, e nenhum deles teve sangramento pósoperatório. A resposta positiva, em curto prazo, após oito semanas do tratamento foi conseguida por $88 \%$ dos pacientes. Conclusões - A esplenectomia laparoscópica é alternativa segura para todas as principais indicações de esplenectomia e pode ser utilizada de forma rotineira.

\section{Correspondência:}

Eduardo Neubarth Trindade

eduardontrindade@yahoo.com.br

Fonte de financiamento: não há

Conflito de interesses: não há

Recebido para publicação: 21/11/2012 Aceito para publicação: 04/02/2013

HEADINGS - Splenectomy. Spleen.
ABSTRACT - Background - Laparoscopic splenectomy is an alternative for the treatment of patients undergoing elective splenectomy. One of its main indications is in hematologic diseases non-responsive to pharmacological treatment. Videolaparoscopy presents advantages to patients when compared to laparotomy: less post-operative pain, recovery of the functions of the gastrointestinal tract, better cosmetic results and shorter hospitalization. Aim - To present a case series of laparoscopic splenectomy in a university hospital. Methods - Were analyzed all the laparoscopic splenectomies between June 2005 and October 2012. The analysis was conducted prospectively divided into pre-, trans-, and post-operative data on: gender, age, indication for surgery, rate of conversion to open surgery, duration of surgery, spleen size, presence of an accessory spleen, time hospitalization and short-term response in eight weeks after the procedure, by analyzing hemoglobin and platelets pre- and post-operative, broken down by gender. Results - Were analyzed 44 laparoscopic splenectomies performed in the period. Patients diagnosed with idiopathic thrombocytopenic purpura accounted for $56.8 \%$, non-responsive to pharmacological treatment; autoimmune hemolytic anemia was $13.6 \%$; spherocytosis, $11.3 \%$ and $18.3 \%$ by other non-hemolytic causes. Six patients had to be converted to open surgery (13.63\%), four due to excessive bleeding. The mean operative time was 166.7 (60-319) minutes and the length of hospitalization was 12 days. Only four patients (9.1\%) had post-operative complications, and none had bleeding after surgery and the positive response in the short term, after eight weeks of treatment, was achieved by $88 \%$ of patients. Conclusions - Laparoscopic splenectomy is a safe alternative for all major indications of splenectomy and can be routinely used. 
INTRODUÇÃO

Esplenectomia laparoscópica (EL) é importante ferramenta no tratamento de doenças hematológicas e outras enfermidades que acometem o baço, e a sua remoção representa terapia efetiva para os pacientes com doença refratária, recorrente ou crônica, nas quais o tratamento medicamentoso falha ou é insuficiente ${ }^{2}$. Desde a década de noventa, a esplenectomia videolaparoscópica vem demonstrando resultados satisfatórios e considerável aumento do número de casos, com consequente padronização da técnica, melhor controle da hemostasia e prevenção da ruptura do órgão, tornando-se não mais uma alternativa, mas sim uma opção terapêutica rotineira para o tratamento das doenças esplênicas ${ }^{2,7}$.

Dentre os benefícios da abordagem laparoscópica estão: menor dor pós-operatória, melhor aparência estética, menores incisões e menor incidência de complicações pós-operatórias, resultando em menor tempo de internação e morbimortalidade; mas, ainda não é considerada a técnica "padrão-ouro" pelos cirurgiões ${ }^{1,2,7}$. Isso se deve principalmente pela redução do trauma durante o acesso esplênico, visão ampliada do campo cirúrgico e à não-manipulação do lado esquerdo do diafragma ${ }^{4}$.

Os resultados positivos demonstrados para as doenças hematológicas, e ainda com advento da cirurgia minimamente invasiva, com sucesso comprovado para colecistectomia e outros procedimentos em relação à técnica aberta, expandiram o uso da EL para doenças esplênicas de outras causas, como esplenomegalia maciça, doenças malignas não-hematológicas e lesão esplênica, não sendo esta mais contraindicação cirúrgica, apesar do risco de ruptura do órgão, sangramento excessivo ou conversão no transoperatório $0^{1,2,7,14}$. Além disso para púrpura trombocitopênica idiopática, hiperesplenismo secundário à cirrose e em casos de trauma esplênico nas situações emergenciais, ela quando comparada à laparotomia apresenta a mesma eficácia, porém com melhores resultados pós-operatórios ${ }^{2,5,14}$.

O objetivo deste estudo foi apresentar uma série de casos para mostrar que a EL pode ser usada como técnica rotineira por alcançar bons resultados pósoperatórios, deixando assim de ser considerada como uma opção terapêutica de exceção.

\section{MÉTODOS}

O projeto de pesquisa foi submetido e aprovado pelo Comitê de Ética em Pesquisa do Hospital de Clínicas de Porto Alegre.

Entre junho de 2005 e outubro de 2012, foram realizadas 44 EL no Hospital de Clínicas de Porto Alegre. A análise foi feita prospectivamente e dividida em pré-, trans- e pós-operatória dos seguintes aspectos: gênero, idade, ASA, indicação cirúrgica, taxa de conversão para laparotomia, tempo cirúrgico, tamanho do baço, presença de baço acessório, tempo de internação e resposta em curto prazo oito semanas após o procedimento através da análise de hemoglobina e plaquetas pré- e pósoperatórias, discriminadas por gênero.

\section{Técnica cirúrgica}

Todos os pacientes foram submetidos à anestesia geral e colocados em decúbito dorsal com coxim sobre o dorso à esquerda. Era introduzido trocarte de $10 \mathrm{~mm}$ e, subsequentemente, a óptica de 300 para visualização do sítio de punção e da cavidade abdominal com vistas à existência de baço acessório. Os procedimentos operatórios foram: a) dissecção do polo inferior do baço com bisturi ultrassônico; b) introdução dos trocárteres na linha média no epigástrio $(10 \mathrm{~mm})$ e a meio do caminho $(5 \mathrm{~mm})$, hipocôndrio esquerdo (12 mm) e flanco esquerdo (5 mm); c) abertura do ligamento gastroesplênico com bisturi ultrassônico; d) ligadura dos vasos gástricos curtos com o mesmo instrumental; e) identificação e ligadura dupla com cliques (hemolock) da artéria esplênica; f) mesmo procedimento com a veia esplênica; g) dissecção da cauda pancreática separando-a do hilo esplênico e liberação de todas as aderências junto ao baço.

\section{RESULTADOS}

Foram realizadas $44 \mathrm{EL}$ no período analisado. Os parâmetros pré-operatórios foram: $77,2 \%$ eram mulheres com média de idade de 35,6 anos (14-68); 36 pacientes foram classificados como ASA II, 5 ASA III e 3 ASA I, e 17 apresentavam comorbidades. As principais indicações cirúrgicas (Tabela 1), foram: 56,8\% tinham diagnóstico de púrpura trombocitopênica idiopática, sem resposta ao tratamento farmacológico; $13,6 \%$ anemia hemolítica auto-imune; $11,3 \%$ esferocitose; $18,3 \%$ outras causas não-hemolíticas. Em $81,8 \%$ dos pacientes havia sido feitoo algum tipo de tratamento clínico sem resposta, principalmente corticoterapia (72,2\%).

TABELA 1 - Principais indicações cirúrgicas

\begin{tabular}{|l|c|}
\hline Doença & $\%$ Número \\
\hline Púrpura trombocitopênica idiopática (PTI) & $56,8 \%(25)$ \\
\hline Anemia hemolítica auto-imune & $13,6 \%(6)$ \\
\hline Esferocitose & $11,3 \%(5)$ \\
\hline Melanoma metastático & $2,27 \%(1)$ \\
\hline Síndrome de Evans & $2,27 \%(1)$ \\
\hline Anemia hemolítica não auto-imune & $2,27 \%(1)$ \\
\hline PTI secundária ao HIV & $2,27 \%(1)$ \\
\hline Linfoma não Hodgkin difuso & $2,27 \%(1)$ \\
\hline Síndrome mielodisplásica & $2,27 \%(1)$ \\
\hline Angiossarcoma & $2,27 \%(1)$ \\
\hline Angiomatose esplênica & $2,27 \%(1)$ \\
\hline
\end{tabular}

Os principais eventos trans-operatórios (Tabela 2) foram: sete pacientes (15,9\%) com baço acessório durante a revisão da cavidade; $13,63 \%$ (6) precisaram 
ser convertidas à laparotomia, quatro por sangramento excessivo, um por esplenomegalia e o outro por controle da hemostasia; $18,2 \%$ (8) pacientes apresentaram sangramento difuso no trans-operatório.

TABELA 2 - Eventos trans-operatórios

\begin{tabular}{|l|c|}
\hline Evento & Número, \% \\
\hline Baço acessório & $7(15,9 \%)$ \\
\hline Conversão à aberta & $6(13,65)$ \\
\hline - Sangramento excessivo & $4(66,6 \%)$ \\
\hline - Esplenomegalia & $1(16,6 \%)$ \\
- Controle da hemostasia & $1(16,6 \%)$ \\
\hline
\end{tabular}

A análise pós-operatória evidenciou necessidade de transfusão para 13,63\% (6) pacientes. Apenas quatro $(9,1 \%)$ apresentaram complicações pós-operatórias (hematoma do músculo reto abdominal, coleção no espaço renal posterior, fístula pancreática e pneumonia nosocomial) e nenhum teve sangramento após a operação; resposta positiva em curto prazo, após oito semanas, foi obtida em $88 \%$ dos pacientes (Tabela 3 ).

TABELA 3 - Dados pós-operatórios

\begin{tabular}{|l|c|}
\hline & Número \% \\
\hline Transfusão & $6(13,63 \%)$ \\
\hline Complicações & $4(9,1 \%)$ \\
\hline - Hematoma reto-abdominal & $1(2,27 \%)$ \\
\hline - Coleção no espaço renal posterior & $1(2,27 \%)$ \\
- Fístula pancreática & $1(2,27 \%)$ \\
\hline - Pneumonia nosocomial & $1(2,27 \%)$ \\
\hline Sangramento & $0(0 \%)$ \\
\hline Resposta positiva em curto prazo & $39(88 \%)$ \\
\hline Recorrência da doença de base & $5(11,3 \%)$ \\
\hline - Anemia hemolítica auto-imune & $3(6,81 \%)$ \\
- Anemia hemolítica não auto-imune & $1(2,27 \%)$ \\
\hline - Síndrome de Evans & $1(2,27 \%)$ \\
\hline
\end{tabular}

A análise laboratorial $(n=43)$ mostrou: média total do índice plaquetário e da hemoglobina no mês antecedente à operação de $114142,86 \mu / L$ (1-321x103) e $11,4 \mathrm{~g} / \mathrm{dL}$ respectivamente. No segundo (não é primeiro em vez de segundo?) mês pós-operatório, 376475 e 12,58g/dL; quando analisados por gênero, a média do índice plaquetário foi $2128875 \mu / L$ para os homens e $8543,75 \mu / L$ para as mulheres, enquanto que os valores de hemoglobina foram $9,8 \mathrm{~g} / \mathrm{dL}$ para homens e $11,8 \mathrm{~g} / \mathrm{dL}$ para mulheres. No segundo mês do pós-operatório foi de $459444,4 \mu$ /L para homens e 3521166,6 para mulheres, enquanto que os valores de hemoglobina foram 12,8 g/dL para homens, e $12,6 \mathrm{~g} /$ dL para mulheres. O peso médio do baço foi de $298,2 \mathrm{~g}$.

\section{DISCUSSÃO}

Os avanços na habilidade e tecnologia têm possibilitado cirurgiões a realizar por via laparoscópica a maioria dos procedimentos realizados por via aberta ${ }^{2,6}$. A primeira descrição de esplenectomia por videolaparoscopia ocorreu em 1991 e, desde então, tem sido favorecida sobre a operação convencional, pois permitiu significativa redução da mortalidade e morbidade. Por isso, o desenvolvimento da técnica laparoscópica tem aumentado o número de esplenectomias realizadas em oposição à terapia médica continuada ${ }^{7}$. Além disso, a EL oferece visualização superior e melhor acesso ao baço, evitando-se grande incisão laparotômica, necessária quando a esplenectomia aberta é realizada ${ }^{10}$.

Esta abordagem cirúrgica é preferida especialmente por pacientes jovens que acham preferíveis as pequenas cicatrizes. É também de grande valor para a gestão de outras lesões benignas e doenças malignas do baço. Além disso, a EL pode ser utilizada com sucesso como opção de tratamento imediato em pacientes hemodinamicamente estáveis com lesão esplênica grave. A abordagem laparoscópica tem a mesma eficácia hemostática da técnica aberta, mas com resultados muito melhores para o paciente ${ }^{7}$.

A esplenectomia laparoscópica apresenta menor morbidade que a aberta e eficácia comparável no tratamento de doenças hematológicas ${ }^{6}$. Vantagens da abordagem laparoscópica quando comparada com a laparotômica incluem a possibilidade de realizar outros procedimentos abdominais concomitantes, tais como colecistectomia, apendicectomia e diverticulectomia de Meckel, sem aumentar as incisões cirúrgicas ${ }^{7}$.

Além disso, recentemente Rescorla et al. ${ }^{11}$ descreveram que a EL resultou em menor custo hospitalar total que a aberta. Ela requer maior investimento em equipamentos, mas, considerando-se que a maioria dos instrumentos é reutilizável, tais custos podem ser reduzidos. A partir dos aprimoramentos das habilidades da equipe cirúrgica, o tempo operatório e a permanência hospitalar são diminuídos, compensando o gasto inicial ${ }^{11}$.

Ainda, os valores do hematócrito parecem se recuperar de forma mais eficaz após a laparoscopia, sugerindo menos hemorragia operatória em relação à aberta ${ }^{12}$. Nessa série, as plaquetas do pré-operatório em relação ao pós-operatório tiveram diferença de $261898,68 \mu / L$ e a diferença da hemoglobina foi de 1,2, mostrando boa evolução. Outro estudo mostrou diminuição na perda sanguínea no trans-operatório; as razões para essa observação são complexas e incluem melhor instrumentação, técnica operatória e experiência do cirurgião aumentada. Dados durante a esplenectomia aberta sugerem que o peso do baço é diretamente proporcional à perda sanguínea, uma vez que quanto maior o tamanho do órgão mais vascularizado é o seu pedículo, exigindo maior cuidado técnico e atenção do cirurgião 3,14,15.

No sentido de minimizar os riscos e aumentar os benefícios da esplenectomia, a cirurgia minimamente invasiva foi introduzida. É esperado que ela elicie resposta neuroendócrina mais modesta no pósoperatório, o que explica a restauração mais rápida da função gastrointestinal ${ }^{12}$. Atualmente, muitos centros têm adotado a EL como opção viável, porém, o manejo 
da esplenomegalia e de baços acessórios ainda gera preocupação9. Mas uma metanálise revelou que a EL pode ser considerada opção aceitável, mesmo em casos de esplenomegalia ${ }^{2}$. O atual estudo teve sete casos de baço acessório, totalizando $15,9 \%$.

A curva de aprendizagem é de grande valor, pois influencia diretamente na taxa de conversão cirúrgica, que diminui sensivelmente com o aumento do número de casos operados, e varia de 0 a 46,7\%8,14. Esse estudo apresentou seis pacientes (14,3\%) que necessitaram de conversão, sendo quatro por sangramento excessivo. A obesidade, doença hematológica e esplenomegalia são fatores predisponentes à conversão ${ }^{6}$. A esplenomegalia é a principal dificuldade técnica na EL. Inicialmente ela era considerada contraindicação, mas a curva de aprendizado e a melhoria da técnica alteraram essa situação9.

As complicações pós-operatórias ocorreram em quatro pacientes $(9,1 \%)$ : hematoma do músculo reto abdominal, coleção no espaço renal posterior, fístula pancreática e pneumonia nosocomial. As taxas de complicações relatadas variam de 0 a $24 \%{ }^{8}$. A principal diferença entre estudos laparoscópicos e a via aberta é o tipo de complicação. As complicações das operações laparoscópicas são menores, como coleções serosas, hematomas intra-abdominais e derrame pleural. Por outro lado, em pacientes submetidos à esplenectomia aberta, a prevalência de complicações graves é maior, mostrando abscesso hepático pós-operatório requerendo drenagem em 3 a 5\%, sangramento e embolia pulmonar 6 .

A mortalidade relatada é menor do que $1 \%$. No presente estudo, a mortalidade foi de três pacientes (6,8\%). Sendo assim, a laparoscopia continua sendo escolha segura para esplenectomia eletiva, com menos complicações e menor tempo de internação no pós-operatório ${ }^{1}$.

\section{CONCLUSÃO}

A técnica videolaparoscópica mostrou-se ser opção segura e eficaz para as esplenectomias eletivas e pode ser utilizada rotineiramente.

\section{REFERÊNCIAS}

1. Andrew-Paul Deeb AP, Kim MJ, Fleming FJ, Messing S, Gunzler D, Monson JRT, Salloum RM. The Impact of Operative Approach in Elective Splenectomy: A Multivariate Analysis of Outcomes From the NSQIP Database. Surg Laparosc Endosc Percutan Tech. 2012; 22: 415-419.

2. Bai $Y N$, Jiang $H$, Panjak P. A Meta-Analysis of Perioperative Outcomes of Laparoscopic Splenectomy for Hematological Disorders. World J Surg. 2012; 36:2349-2358.

3. Bell RL, Reinhardt KE, Cho E, Flowers JL. A ten-year, single institution experience with laparosco splenectomy. JSLS. 2005; 9:163-168.

4. Bhandarkar DS, Katara AN, Mittal G, Rasik Shah R, Udwadia TE. Prevention and Management of Complications of Laparoscopic Splenectomy. Indian J Surg. 2011; 73(5): 324-330.

5. Carobbi A, Romagnanin F, Antonelli G, Bianchini M. Laparoscopic splenectomy for severe blunt trauma: initial experience of ten consecutive cases with a fast hemostatic technique. Surg Endosc. 2010; 24:1325-1330.

6. Coelho JCU, Claus CMP, Bombana B, Machuca TN, Sobottka WH. Esplenectomia laparoscópica. Rev Col Bras Cir. 2004; 31(3):200-203.

7. Corcione F, Pirozzi F, Aragiusto G, Galante F, Sciuto A. Laparoscopic splenectomy: experience of a single center in a series of 300 cases. Surg Endosc. 2012; 26:2870-2876.

8. Katkhouda N, Mavor E. Splenectomy laparoscopic. Surg Clin North Am. 2000; 80(4): 1285-1297.

9. Melo-Filho AA, Miranda ML, Oliveira-Filho AG, Pinheiro $V R$, Brandalise NA, Bustorff-Silva JM. Esplenectomia laparoscópica nas doenças hematológicas. Rev Col Bras Cir. 2003; 30(5): 382-387.

10. Park A, Targarona EM, Trias M. Laparoscopic surgery of the slpeen: state of the art. Langenbeck's Arch Surg. 2001; 386:230-239.

11. Rescorla FJ, Breitfeld PP, West KW, ET al. A case controlled comparison of open and laparoscopic splenectomy in children. Surgery. 1998; 124:670-676.

12. Sapucahy MV, Faintuch J, Bresciani CJC, Bertevello PL,Habr-Gama A, Gama-Rodrigues JJ. Laparoscopic versus open splenectomy in the management of hematologic diseases. Rev Hosp Clin Fac Med S. Paulo. 2003; 58(5):243-249

13. Trindade MR, Trindade EN, Boza JC, Mottin M, Mossmann DF, Von Diemen V. Esplenectomia videolaparoscópica em pacientes com doenças hematológicas. Rev AMRIGS. 2007; 51: 67-68.

14.Zhou J, Wu Z, Panja P, Peng B. Long-term postoperative outcomes of hypersplenism: laparoscopic versus open splenectomy secondary to liver cirrhosis. Surg Endosc. 2012; DOI 10.1007/ s00464-012-2349-6. 